\title{
VIDEOGAME: É DO BEM OU DO MAL? COMO ORIENTAR PAIS
}

\author{
Luciana Alves* \\ Alysson Massote Carvalho"
}

\begin{abstract}
RESUMO. Desde a década de 70 milhares de crianças, adolescentes e adultos praticam jogos de computador ou videogame. O objetivo deste artigo foi identificar os efeitos deste artefato tecnológico para a cognição, sua aplicabilidade e sua influência sobre o comportamento e a saúde do jogador e, desta forma, delinear orientações para pais e profissionais de saúde que lidam com crianças e adolescentes. Na primeira parte do artigo são apresentados alguns efeitos negativos e positivos dos jogos; na segunda é proposto um conjunto de orientações que podem tornar a brincadeira mais segura; e na última parte, as considerações finais. Concluiu-se que o videogame é uma ferramenta capaz de aperfeiçoar as habilidades cognitivas e perceptivas, sendo os jogos educacionais apontados como uma experiência benéfica aos seus usuários, e que as repercussões negativas se referem, principalmente, ao efeito daqueles com conteúdo de violência.
\end{abstract}

Palavras-chave: Videogame, saúde, adolescentes.

\section{VIDEOGAME: IS IT FROM THE GOOD OR THE EVIL? HOW TO ORIENT PARENTS}

\begin{abstract}
Since the 70's thousands of children, young people and adults play computer or videogame games. This study aimed to identify the effects of this technological device on cognition, its applicability and influence on player behavior and health and to establish some guidelines for parents and professionals of health that work with children and adolescents. In the first part we presented some positive and negative effects of the games. In the second part it is proposed a set of orientations in order to keep safety and the final considerations are in the last part. We conclude that videogame is an effective tool for cognitive and perceptive skills optimizing. The educational games are identified as a beneficial experience for its users and negative repercussions are found in those with violence content.
\end{abstract}

Key words: Video game, health, adolescents.

\section{¿ES BUENO O MALO? CÓMO ACONSEJAR A LOS PADRES}

RESUMEN. Desde los años 70, miles de niños, adolescentes y adultos juegan juegos de computadora o videojuegos. La finalidad de este trabajo fue identificar los efectos de este artefacto tecnológico a la cognición, su aplicabilidad, influencia para el comportamiento y la salud del jugador y así elaborar directrices para los padres y los profesionales de la salud que trabajan con niños y adolescentes. En la primera parte del artículo se presentan algunos efectos negativos y positivos de los juegos. En la segunda, se propone un conjunto de directrices que pueden hacer el juego más seguro, y en la última expone las consideraciones finales. Se concluyó que los videojuegos son herramientas que pueden mejorar las aptitudes cognitivas y perceptivas, donde los juegos educativos son señalados como una buena influencia para aquellos que los practican y por otra parte aquellos con contenido de violencia son evaluados negativamente por la mala influencia.

Palabras-clave: Videojuegos, salud, adolescentes.

Historicamente, a criação do primeiro videogame ou jogos de computador data de 1958 e se deve ao físico Willy Higinbotham, um dos cientistas que colaboraram para a invenção da bomba atômica. Em 1968 Ralph Baer patenteou o primeiro videogame. A partir daí, desde a criação da Atary, na década de 70, por Nollan Busnell e Ted Dabney, a indústria desse artefato não parou de crescer (Reis, 2005).

Diante deste visível crescimento dos jogos como meio de diversão, há uma crescente preocupação em

Psicóloga, Doutora em Ciências da Saúde pela Faculdade de Medicina da Universidade Federal de Minas Gerais-UFMG.

\# Pós Doutor em Psicologia pela UNCG. Orientador do Programa de Pós-graduação em Ciências da Saúde, Saúde da Criança e do Adolescente da Faculdade de Medicina da UFMG. Diretor Geral do Instituto Presbiteriano Gammon. 
realizar estudos empíricos sobre este assunto. No Brasil, a literatura indexada aborda de forma incipiente o tema e não temos ainda um know-how em pesquisas com este enfoque.

Para elencar um conjunto de recomendações com vista a subsidiar os profissionais de saúde em suas orientações aos pais e à sociedade quanto ao uso saudável de jogos de videogame, buscamos compreender o impacto do uso do videogame sobre a cognição e sobre a própria sociedade. Neste sentido foram feitas pesquisas nas bases de dados LILACS, MEDLINE e SciELO na Biblioteca Virtual de Saúde (BVS) e PubMed, além de busca, no Google Acadêmico, por artigos citados no material coletado. Os termos utilizados para busca na BVS foram: "Video game", "Videogame". No aceso à PubMed os termos utilizados foram: "ídeo game" ou "computer game" ou "videogame", até o ano de 2008.

Organizamos o presente artigo em três partes. $\mathrm{Na}$ primeira abordaremos alguns aspectos negativos e positivos dos jogos, como também algumas aplicações dos jogos de videogame; na segunda descreveremos um conjunto de diretrizes para os profissionais orientarem os pais e crianças no sentido de terem uma relação mais saudável e segura com os jogos; e na parte final exporemos nossas conclusões. Não serão abordados os jogos de realidade virtual (RV).

\section{EFEITOS NEGATIVOS GERAIS DOS JOGOS DE VIDEOGAME}

O aumento da violência e dos níveis de agressividade por meios midiáticos com conteúdos violentos no cotidiano dos jovens tem sido foco de muitos estudos. Embora os jogos de videogame tenham emergido nos anos de 1970, foi somente na década de 1990 que os jogos violentos entraram em cena, com Wolfenstein e Mortal Kombat. Figura entre os aspectos negativos dos jogos seu efeito sobre o comportamento, a cognição e a saúde.

Pesquisas revelaram que os jogos violentos podem levar a comportamentos agressivos e à delinquência . Segundo Uhlmann e Swanson (2004), determinados jogos com conteúdo de violência podem levar o adolescente a perceber-se como agressivo. Em seu estudo, adolescentes que dedicavam muito de seu tempo a jogos de videogame com conteúdo de violência foram considerados mais hostis, discutiam com professores mais frequentemente, tinham maior probabilidade de se envolver em lutas físicas e pior desempenho escolar (Gentile, Lynch, Linder \& Walsh, 2004). A prática repetida de jogos violentos aumentou os comportamentos agressivos em função da mudança nos fatores cognitivos e de personalidade (Bartholow, Sestir \& Davis; 2005).

A dependência ou vício de jogos é outro aspecto que motiva investigações. Em revisão sistemática com enfoque na dependência de internet e de jogos eletrônicos, as análises revelaram que as prevalências apontadas em diferentes estudos são muito diferentes entre si, o que poderia ser delinquência das diferentes nomenclaturas utilizadas nas referências a este problema. Este fato levaria também à adoção de diferentes critérios diagnósticos para o problema. Os autores concluem serem necessárias mais investigações sobre este transtorno e, talvez, uma nova classificação psiquiátrica para o século XXI, e levantam a questão de que o uso abusivo de jogos eletrônicos e de internet podem ser parte de outros transtornos já descritos na literatura psiquiátrica (Abreu, Karam, Góes \& Spitzer; 2008).

Os aspectos da saúde que podem ser afetados pelo uso do videogame são vários, porém a literatura da área ainda é reduzida e outros estudos devem ser feitos para esclarecer melhor os mecanismos subjacentes a cada uma das entidades nosológicas e sua associação com o videogame. A seguir descrevemos alguns achados.

\section{Epilepsia}

Ataques epilépticos causados por jogar videogame têm sido reportados pela literatura da área da neurologia desde a década de 50, mas a popularização do uso do videogame tornou-a um fato mais comum. As crises epilépticas podem ser desencadeadas pelo piscar rápido e rítmico de luzes, que gera descargas elétricas anormais no cérebro.

Embora estes ataques não sejam uma novidade, sua natureza e o elo existente entre eles e os vários tipos de jogos são incertos. Muitos autores consideram que os ataques induzidos pelos jogos de videogame sejam uma extensão da epilepsia fotossensível, enquanto outros relatórios apontam para algum outro mecanismo que não este da fotossensibilidade em relação aos ataques provocados pelos jogos de videogame. Em um estudo foram investigados dois grupos. O grupo I era formado por 10 pacientes cujos ataques haviam surgido quando jogavam ou assistiam a um jogo específico; e o grupo II constituía-se de 12 pacientes cujos ataques haviam surgido tanto quando eles jogavam quanto de modo espontâneo. No grupo I foram encontradas anormalidades não específicas em $60 \%$ dos eletroencefalogramas (EEG) ou imagens de ressonância cerebral, e um ataque parcial foi reconhecido em $30 \%$ dos pacientes. No grupo II a maioria apresentava ataques tônico-clônicos, ataques 
mioclôncos e ausências, e 42\% apresentaram descargas epileptiformes no EEG. Estes autores concluíram que os ataques relacionados aos jogos não são uma síndrome rara e homogênea e que pode haver diferentes mecanismos relacionados à eclosão da crise. Também concluíram que a melhor medida preventiva é evitar estes tipos de jogos (Chuang et al., 2006).

\section{Síndromes musculoesqueléticas}

Conquanto o uso excessivo de computadores ou videogames possa levar a problemas musculoesqueléticos, em um estudo realizado na cidade de São Paulo por Zapata, Moraes, Leone, Doria-Filho e Silva (2006) em uma população de 833 adolescentes, os resultados demonstraram que o uso frequente de computador ou videogame não estava associado com a presença de dor e síndromes de dor musculoesqueléticas.

\section{Sono}

Segundo Higuchi, Motohashi, Liu e Maeda (2005), alguns estudos epidemiológicos mostram que jogar videogame à noite pode atrasar a hora de dormir e encurtar as horas dormidas, porém os efeitos sobre a arquitetura do sono e sua qualidade ainda não estão claros. Em uma pesquisa foram avaliados em laboratório os efeitos de jogar videogame sobre o sono noturno. Os sujeitos, sete homens adultos, foram seus próprios controles, e, em resumo, os pesquisadores concluíram que jogar videogames estimulantes antes de dormir levou a um menor autorrelato de sonolência antes de ir para a cama, aumentou a latência do sono e diminuiu o sono REM em relação à situação controle.

\section{Doença mental}

Um estudo relatado por Forsyth, Harland e Edwards (2001) descreveu um caso de esquizofrenia em um homem jovem após jogar videogame, cujos sintomas se desenvolveram por um período de dois anos. $\mathrm{Na}$ entrevista o jovem relatava ter a ilusão de que era um jogador dentro de um jogo de computador em que os pontos eram marcados a partir do roubo de carros, com mortes e fugas de carros de polícia. Segundo o relato do rapaz, este sentia que os jogos se comunicavam com ele por meio dos fones de ouvido. Para ganhar pontos, ele começou a roubar carros e assaltar com armas. Também relatou que não teria pesar em matar, desde que isto aumentasse sua pontuação.

Estes autores ressaltam não quererem dizer com isto que jogos de videogame possam causar psicose, contudo alertam sobre a possibilidade de os cenários de jogos serem incorporados crescentemente ao sistema de ilusão.

\section{Metabolismo}

Existe um crescente interesse de profissionais da saúde pelos efeitos de estilos de vida sedentários em jovens, particularmente naqueles com sobrepeso ou obesidade. Wang e Perry (2006) examinaram respostas metabólicas, fisiológicas e hemodinâmicas em crianças que jogavam um videogame de ação denominado "Tekken 3" e nas quais foram controlados a pressão arterial antes e ao longo do jogo, a glicose sanguínea e os níveis de lactato antes e após o jogo. Neste estudo foi encontrado um aumento da pressão sanguínea sistólica e diastólica, do batimento cardíaco, da ventilação, do consumo de oxigênio, do índice respiratório e do gasto de energia. O presente estudo demonstrou que, embora o jogo de videogame não seja uma atividade sem efeito para crianças jovens, já que resulta em aumentos significativos em várias respostas fisiológicas e metabólicas, a intensidade desses realces é insuficiente para uma melhora do condicionamento cardiovascular, uma vez que as mudanças ocorridas nas variáveis medidas eram mais baixas do que aquelas associadas com exercício físico.

Em estudo realizado por Hérbert, Béland, DioneFournelle, Crête e Lupien (2005) foi investigado, em jogadores de videogame, o papel do som na liberação do hormônio do estresse, particularmente do cortisol, a partir da análise da saliva. A principal hipótese investigada foi a de que a música dos jogos de videogame seja a maior fonte de estresse nos jogadores. Os resultados mostraram que os níveis de cortisol após coletas seriadas foram significativamente mais elevados no grupo que jogou com música em relação ao grupo na presença de silêncio.

\section{EFEITOS POSITIVOS GERAIS DOS JOGOS DE VIDEOGAME}

Quando focamos os aspectos positivos dos jogos, geralmente os jogos educacionais são aqueles que primeiro vêm à mente de quem discute o assunto.

Goodman, Bradley, Paras, Williamson e Bizzochi (2006) desenvolveram um jogo de videogame para jogadores de hockey e constataram a eficiência do uso do jogo para ensinamentos sobre concussão. Kato e Beale (2006) investigaram a aceitabilidade de um jogo de ação sobre câncer como uma ferramenta de aprendizagem a respeito da doença e dos cuidados durante o tratamento. Concluíram, no que se refere ao 
câncer, que o jogo de videogame talvez seja uma ferramenta útil para melhorar o entendimento destes jovens sobre essa doença e o autocuidado durante seu tratamento. Yoon e Godwin (2007) também constataram que um game educacional desenvolvido especificamente para crianças com anemia falciforme resultou na ampliação do conhecimento destas crianças sobre a doença e no gerenciamento de seus sintomas, aumentando sua confiança na aplicação deste conhecimento.

Em um estudo longitudinal de Durkin e Barber (2002) que investigou 1304 adolescentes cujo envolvimento com jogos era graduado em "nunca", "baixo" ou "alto", concluiu-se não haver evidências em várias medidas (saúde metal, proximidade familiar, uso de substâncias, autoconceito, rede de amizade, desobediência aos pais) de que jogadores de videogame se saiam pior nestes quesitos, inclusive no desempenho escolar. Além disso, os autores ressaltam que esses jogadores, principalmente os que jogam com "baixa" frequência, obtiveram resultados mais favoráveis do que os não jogadores. Em outro estudo envolvendo 4.508 adolescentes, realizado por Sharlf e Sargent (2006), os autores correlacionaram a performance escolar com as variáveis tempo despedido com TV e jogos de videogame, disponibilidade de canais de TV a cabo e o grau de policiamento dos pais em relação aos programas de TV e filmes assistidos. Estes autores concluíram que não existe correlação entre videogame e desempenho escolar, mas recomendam que o tempo despedido com jogos de videogame seja em torno de uma hora.

Em 2003, Green e Bavelier publicaram o resultado de uma pesquisa com uma população de jovens incluindo jogadores de videogame (JVGs) e não jogadores de videogame (NJVGs). Os grupos foram testados nas tarefas habilidade de ignorar um distrator, enumeração, distribuição espacial da visão e Attentional blink. O grupo de NJVGs foi dividido em dois grupos e treinado em dois jogos, Tretis e Medal of Honor (um videogame de ação) e retestado posteriormente. Neste estudo, na situação pré-teste, concluíram que os jogos de videogame aumentam a capacidade do sistema de atenção visual dos jogadores e que estes devem exaurir seus recursos de atenção visual mais lentamente que NJVGs. Concluíram também que jogar videogame melhora a atenção visual e sua distribuição espacial. Na tarefa de enumeração, os JVGs identificaram mais itens e o fizeram mais imediatamente que os NJVGs, e os JVGs foram significativamente mais acurados que os NJVGs; e o tempo de processamento e informação visual foi menor em JVGs.
A análise dos resultados e das conclusões póstreinamento do grupo de NJVG revelou que, no Attentional blink task e na tarefa de enumeração, o grupo que jogou Medal of Honor teve um desempenho superior ao daquele que não jogou. Os pesquisadores concluíram que jogar videogame pode alterar significativamente o processo de atenção visual. No que tange à distribuição da atenção visual, o grupo treinado no videogame de ação melhorou significativamente em relação ao pré-teste, mais do que o dos que jogaram Tretis (controle).

Estas mesmas pesquisadoras, em 2006, publicaram estudo em que concluíram, por meio de cinco experimentos, que os jogos de videogame de ação aumentam o número de objetos que podem ser enumerados e seguidos simultaneamente quanto ao tempo. JVGs de ação são mais acurados na enumeração de itens do que não jogadores, mas, apesar disso, estes jogos não modificam o número de itens que podem ser imediatamente apreendidos, o que melhora é o número de itens que se podem contar de forma exata.

Castel, Pratt e Drummond (2005) investigaram como os jogos de videogame de ação podem influenciar a atenção seletiva visual. Para isso examinaram semelhanças e diferenças entre JVGs e NJVGs nas habilidades de inibir a atenção para retornar a situações previamente conhecidas e de eficácia na procura visual. Foi utilizado um paradigma denominado de inibição do retorno conhecido como inhibition of return (IOR). Trata-se de uma preferência da atenção por locais não conhecidos e inesperados em relação àqueles que a pessoa já experienciou. Os resultados confirmaram que existem diferenças claras entre JVGs e NJVGs em tarefas visuais de atenção.

Em 2006, Nagamitsu, Nagano, Yamashita, Takashima e Matsuishi investigaram o volume sanguíneo cerebral em jogadores de videogame. Os resultados apontaram várias mudanças nas concentrações de hemoglobina $(\mathrm{Hb})$ durante a prática do jogo de videogame Donkey Kong em três diferentes posições do córtex pré-frontal e dos frontoparietais dos pesquisados, tanto nas crianças quanto nos adultos. Concluíram que estas mudanças talvez sejam determinadas pelos fatores desempenho, níveis de atenção e interesse, respostas fisiológicas ou utilização de idade relacionada de circuitos neurais diferentes. Neste mesmo ano, Matsuda e Hiraki (2006) publicaram um estudo onde analisaram as mudanças na oxyHb como o melhor indicador de atividade cerebral em crianças. Estas jogaram Melee (um jogo de luta) e Tetris (um quebra-cabeça). Os resultados 
mostraram ter havido mudanças hemodinâmicas da concentração de oxyHb durante cada um dos jogos em quatro regiões cerebrais: anterior esquerda, anterior direita, posterior direita e posterior esquerda. Segundo os pesquisadores, provavelmente esta resposta hemodinâmica se deveria a uma exigência de atenção para a prática dos jogos de videogame, e não a questões relacionadas à idade ou ao desempenho.

Quaiser-Pohl, Geiser e Lehmann (2006) investigaram até que ponto a preferência por jogos de videogame se relaciona ao desempenho em uma tarefa de rotação mental (TRM) e a diferenças de gênero. Constataram que os homens se saíram melhor que as mulheres na TRM e os homens NJVGs tiveram pior desempenho na TRM que aqueles que jogavam jogos de ação e simulação. Constataram também que os jogos preferidos pelas mulheres não apresentaram efeitos positivos em relação ao desempenho na TRM.

Embora durante muito tempo se tenha acreditado que as mulheres apresentam menor desenvolvimento nas habilidades espaciais do que os homens, constata-se hoje que os jogos de computador podem minimizar essas diferenças, melhorando habilidades espaciais em ambos os sexos. Feng, Spence e Pratt (2007) realizaram estudo investigando JVGs e NJVGs, como também a diferença de gênero e campo de estudo (Artes e Ciências). No primeiro experimento foram dadas uma tarefa que avaliava o campo útil de visão e uma de rotação mental. Os resultados indicaram um melhor desempenho dos JVGs em relação aos NJVGs. Estes achados são semelhantes ao de Green e Bavelier (2003). No experimento dois, foram comparadas a atenção e a cognição espacial de homens e mulheres considerados NJVGs, antes e depois de um treinamento de 10 horas em um jogo de ação denominado Medal of Honor: Pacific Assault. O grupo controle jogou outro jogo não considerado de ação. Após o treino os grupos foram re-testados. Os resultados apontaram o potencial dos jogos de videogame de melhorar o desempenho de homens e mulheres em tarefas espaciais. Além disso, as mulheres apresentaram uma melhora nesta habilidade superior à dos homens. Diferenças prévias de gênero foram praticamente eliminadas na tarefa de campo de visão útil e reduzidas na tarefa de rotação mental.

Green e Bavelier (2007), usando um paradigma denominado crowding, mostram que o jogo de videogame de ação altera a resolução espacial da visão. Dá-se o nome de crowding à região ou zona de interação espacial ao redor de um alvo onde a presença dos objetos distratores leva a uma diminuição da sensibilidade para o alvo. As pesquisadoras deram como exemplo que, na vida diária, o crowding limita o desempenho na habilidade de identificar letras ou palavras encaixadas no texto.

No primeiro experimento foi medido o tamanho da região do crowding dos JGVs e NJVGs por meio de um teste de sua habilidade em discriminar duas formas de distratores: aqueles apresentados acima e os apresentados abaixo do alvo. O tamanho da região do crowding foi avaliado em três excentricidades diferentes: $0^{\circ}, 10^{\circ}$, e $25^{\circ}$. Os resultados apontaram um melhor desempenho do grupo de JVGs em relação aos NJVGs em todas as excentricidades testadas. No segundo experimento usou-se o paradigma do treinamento com o objetivo de investigar se existe uma relação causal entre a experiência com jogos de videogame de ação e o realce da resolução espacial. Os NJVGs foram divididos em dois grupos de treinamento. Os resultados apontaram que os sujeitos treinados no jogo de ação (Unreal Tournament) aumentaram significativamente sua capacidade de diminuir o tamanho da região de crowding em relação àqueles do grupo controle (Tretis). Assim foi possível estabelecer uma relação causal entre a exposição ao videogame e a redução no tamanho da região de crowding.

Segundo Stickgold, Malia, Maguire, Roddenberry e O'Connor (2000), pessoas que se empenham em atividades físicas ou mentais durante grandes períodos de tempo, frequentemente experimentam uma repetição alucinatória destas atividades no início do sono. Foram estudados três grupos: sujeitos sem experiência com Tetris, sujeitos que tinham experiência considerável neste jogo, mas o não haviam praticado recentemente, e amnésticos com danos bilaterais no lobo temporal medial. Os participantes noviços que jogaram Tretis informaram imagens visuais intrusivas estereotipadas do jogo. Pacientes amnésticos fizeram relato hipnagógico semelhante, embora não pudessem lembrar-se do jogo. Os resultados apontaram que tal imaginação poderia surgir independentemente do sistema de memória declarativa. Os participantes do grupo com experiência no jogo também informaram imagens de jogos previamente jogados, o que pode indicar que talvez memórias remotas influenciem estas imagens. Em outro estudo os pesquisadores também sugeriram que jogos de videogame de ação 
podem melhorar aspectos da memória de trabalho visual (Green \& Bavelier, 2006).

A literatura mostra que os efeitos positivos e a aplicabilidade dos jogos se dão de forma exitosa em diferentes contextos.

Segundo Rosser et al. (2007), uma das profissões que podem obter vantagem por meio do treino com videogame é a de cirurgião laparoscópico. Os pesquisadores relataram que os cirurgiões que faziam uso do videogame por mais de três horas por semana cometiam $37 \%$ menos erros durante a cirurgia e eram $27 \%$ mais rápidos em suas habilidades laparoscópicas, e que $33 \%$ deles eram melhores em testes de sutura, se comparados a colegas que não jogavam videogame.

Os jogos também são apontados como detentores de potencial terapêutico.

Patel et al. (2006) investigaram 112 crianças e constataram que o uso do videogame diminuiu a ansiedade das crianças do grupo que jogou videogame no período pré-operatório e durante a indução anestésica.

Os jogos também têm sido úteis para aumentar o grau de motivação para atividades físicas.

Widman, McDonald e Abresch (2006) encontraram em um grupo de crianças com espinha bífida uma maior motivação para a prática de atividade física, utilizando para isto um game denominado GameCycle.

Os benefícios dos jogos não param por aqui. Segundo outros autores, os jogos têm o potencial de aumentar a socialização entre os jovens.

Fortim (2006) pesquisou por que jovens passavam muito tempo on-line jogando MUD, um jogo de aventura baseado em descrições textuais, com tema medieval, que envolve monstros e um tesouro. Seus achados apontaram que uma das motivações para o jogo era a possibilidade de "sociabilidade" por ele proporcionada. Em outro estudo, Cummings e Vandewater (2007), investigando 1491 adolescentes, não encontraram diferenças no tempo gasto com familiares e amigos, achados que não corroboram a ideia de que adolescentes jogadores de videogame são socialmente isolados.

Ainda se fazem necessários mais avanços na investigação sobre o impacto dos jogos sobre a cognição e comportamentos humanos, em função da complexidade de ambos os focos de investigação, o homem e os jogos de videogame.

\section{DIRETRIZES PARA TORNAR A BRINCADEIRA MAIS SEGURA}

Enquanto a investigação científica envolvendo os jogos levanta questões a respeito de seus efeitos sobre a cognição e o comportamento humanos, pais e profissionais procuram encontrar o melhor caminho para lidar com os jogos e seu fascínio sobre crianças e adolescentes, e com os inevitáveis conflitos que daí advêm. Hoje, o melhor argumento favorável ou contrário ao uso dos jogos envolve bom senso, alguns achados das pesquisas já realizadas e orientações multidisciplinares, como:

- evitar jogar logo antes de dormir;

- evitar jogos não adequados à faixa etária (os jogos trazem a classificação dos jogos em seus respectivos invólucros);

- se os pais julgarem que um jogo não tem um conteúdo adequado para o(a) filho(a), orientá-lo(a) a jogar com eles e a trocar vídeo sobre o tema;

- orientar os pais a procurarem saber quais jogos seus filhos gostam de jogar para saberem a quais conteúdos eles(as) podem estar expostos(as), embora não haja como controlar em $100 \%$ a que tipos de jogos eles vão se expor;

- evitar jogar mais de 50 minutos seguidos sem fazer uma pausa para alongamento de pelo menos 10 minutos a cada 50 minutos, pois alongamentos ajudam a prevenir lesões musculoesqueléticas;

- evitar o uso de headphone ao jogar, ou deixar o som baixo, pois a audição pode ser prejudicada caso o som esteja alto; o ideal é que a altura do som permita que se escute quem está ao lado;

- procurar manter uma distância mínima de $60 \mathrm{~cm}$ da tela do computador, para evitar problemas de visão como a síndrome visual relacionada a computadores - SVRC;

- variar as categorias de jogos, testando diferentes jogos, pode levar o jogador a descobrir um mundo novo e assim desenvolver tipos diferentes de habilidades.

Além disso, é preciso levar em conta que:

- pessoas com histórico de epilepsia devem evitar jogar;

- como alguns jogos demandam tempo superior ao recomendado (cerca de uma hora), é importante ter alguma flexibilidade quanto ao tempo; jogos que demandam mais tempo devem ser praticados nos finais de semana ou nos feriados;

- os filtros protetores auxiliam em muito, contribuindo para a diminuição dos espaços luminosos e aumentando o contraste entre as imagens. 
- a posição do monitor deve estar entre 10 e 20 graus abaixo do nível dos olhos, pois isto proporciona maior conforto postural.

\section{CONSIDERAÇÕES FINAIS}

$\mathrm{O}$ presente artigo investigou, a partir de um levantamento na literatura, possíveis efeitos dos jogos de videogame sobre a cognição, o comportamento humano e sua aplicação em alguns segmentos da sociedade. Foi possível perceber que os jogos considerados violentos podem levar a efeitos até certo ponto negativos, mas é preciso levar em consideração que o universo daquele que joga é composto por outros elementos que fazem parte da arquitetura da vida social. Sternheimer (2007), numa perspectiva sociológica, corrobora esta observação e coloca que culpar os videogames pelos comportamentos violentos é desconsiderar um conjunto de outras variáveis, afirmando a complexidade do assunto.

Neste contexto, muito ainda deve ser esclarecido a respeito deste potencial efeito sobre a cognição e comportamento. A complexidade do tema abordado neste trabalho é tamanha que até mesmo os jogos de caráter não violento podem impactar o sujeito de forma negativa, por se tornarem um possível vício ou levarem à dependência. As repercussões sobre a saúde do indivíduo também ocupam uma longa lista de itens. Alguns jogos, sobretudo os jogos educacionais, são cotados como aqueles potencialmente capazes de gerar experiências benéficas aos seus usuários, como, por exemplo, a melhora na habilidade espacial, a atenção, a motivação e outros. Contrariando a tradicional crença de que os jogos de computador afetam de forma negativa o desempenho escolar, pesquisas mais recentes nos mostram que a prática do videogame pode ser benéfica. Estes benefícios também se estendem ao campo das relações pessoais (amigos e família).

Pesquisas com videogame provaram que este é uma ferramenta capaz de redefinir e aperfeiçoar as habilidades visual-motoras, espacial e de atenção visual.

As pesquisas realizadas sobre videogame, em termos de teoria, estão desvendando um campo fantástico, tanto que a capacidade do cérebro e o comportamento humano podem vir a ser reformulados por meio do treinamento com este artefato. Não obstante, o entendimento dos mecanismos que levam a tamanha plasticidade se constitui, na atualidade, em um dos grandes desafios enfrentados por este campo da pesquisa científica.
As dificuldades que se apresentam ao pesquisador que investiga os efeitos deste artefato tecnológico são inúmeras. Por exemplo, são muitos os tipos de jogos e isso dificulta a comparação dos efeitos cognitivos e perceptivos e a possibilidade de estabelecer de antemão qual jogo modificaria estes aspectos $\mathrm{e}$ as questões relacionadas a gênero ou idade. As dificuldades também se fazem perceber na prática clínica de muitos profissionais, que precisam responder a uma pergunta cada vez mais constante dos pais e da sociedade em geral: qual o limite entre o saudável e o patológico quando o assunto é videogame?

\section{REFERÊNCIAS}

Abreu, C. N., Karam, R.G., Góes, D. S., \& Spitzer D.T (2008). Dependência de internet e de jogos eletrônicos: uma revisão. Rev Bras Psiquiat, 30(2):156-67.

Bartholow, B. D, Sestir, M. A., \& Davis, E. B. (2005). Correlates and consequences of exposure to video game violence: hostile personality, empathy, and aggressive behavior. Personality and Social Psychology Bulletin, 31(11), 1573-1586.

Castel, A. D., Pratt, J., \& Drummond, E. (2005). The effects of action video game experience on time course of inhibition of return and efficiency of visual search. Acta Psychologica, 119, 217-230.

Chuang, Y-C., Chang, W-N., Lin, T-K., Lu, C-H., Chen, S-D., \& Huang, C-R. (2006). Game-related seizures presenting with two types of clinical features. Addiction, 15, 98-105.

Cummings, H. M., \& Vandewater, E. A. (2007). Relation of adolescent ídeo game play to time spent in other activities. Arch Pediatr Adolesc Med., 161(7), 684-689.

Durkin, K., \& Barber, B. (2002). Not so doomed: computer game play and positive adolescent development. Journal of Applied Developmental Psychology, 23, 373-392.

Feng, J., Spence, I., \& Pratt, J. (2007). Playing na action ídeo game reduces gender differences in spatial cognition. Psychological Science, 18(10), 850-855.

Forsyth, R., Harland, R., \& Edwards, T. (2001). Computer game delusions. J R Soc Med, 94, 184-185.

Fortim, I. (2006). Alice no país do espelho: o MUD - o jogo e a realidade virtual baseados em texto. Imaginario, 12(12), 171-194.

Gentile, D. A., Lynch, P. J., Linder, J. R., \& Walsh, D.A. (2004). The effects of violent ídeo game habits on adolescent hostility, aggressive behaviors, and school performance. International journal of adolescence and youth, 27(1), 5-22.

Goodman, D., Bradley, N. L., Paras, B., Williamson, I. J., \& Bizzochi, J. (2006). Video gaming promotes concussion knowledge acquisition in youth hockey players. Journal of adolescence, 29, 351-360.

Green, C. S., \& Bavelier, D. (2003) Action Video game modifies visual selective attention. Nature, 423,534-537.

Green, C. S., \& Bavelier, D. (2006). Enumeration versus multiple object tracking: the case of action video game players. Cognition, 101, 217-245. 
Green, C. S., \& Bavelier, D. (2007). Action-video-game experience alters the spatial resolution of vision. Psychological Science, $18(1), 88-94$.

Hérbert, S., Béland, R., Dione-Fournelle, O., Crête, M., \& Lupien, S. J. (2005) Physiological stress response to video-game playing: the contribution of built-in music. Life Science, 76, 2371-2380.

Higuchi, S., Motohashi, Y., Liu, Y., \& Maeda, A. (2005). Effects of playing a computer game using a bright display on presleeep physiological variables, sleep latency, slow wave sleep and REM sleep. J. Sleep Res., 14, 267-273.

Kato, P. M., \& Beale, I. L. (2006). Factors affecting acceptability to yong cancer patients of a psychoeducational ídeo game about ídeo. Journal of Pediatric Oncology Nursing, 23, 269-275.

Matsuda, G., \& Hiraki, K. (2006). Susteined decrease in oxygenated hemoglobin during video games in the dorsal prefrontal ídeo: A NIRS study of children. Neuroimage, 29, 706-711.

Nagamitsu, S., Nagano, M., Yamashita, Y., Takashima, S., \& Matsuishi, T. (2006). Prefrontal blood volume patterns while playing ídeo games - A near-infrared spectroscopy study. Brain \& Development, 28, 315-321.

Patel, A., Schieble, T., Davison, M., Tran, M. C. J., Schoenberg, C., Delphin, E., \& Benntte, H. (2006). Distraction with a hand-held ídeo game reduces pediatric preoperative anxiety. Pediatric Anesthesia, 16, 1019-1027.

Quaiser-Pohl, C., Geiser, C., \& Lehmann, W. (2006). The relationship between computer-game preference, gender, and mental-rotation ability. Personality and Individual Differences, 40(3), 609-619.

Reis, G. (2005). Videogame: história, gêneros e diálogo com o cinema. Dissertação de Mestrado, Programa de Pós-Graduação da Faculdade de Comunicação, Educação e Turismo, Universidade de Marília, São Paulo. Recuperado em 22 de julho de 2007 de http://www.dominiopublico.gov.br

Rosser, J. C., Lynch, P. J., Cuddihy, L., Gentile, D. A., Klonsky, J., \& Merrell, R. (2007). The impact of vídeo games on train surgeons in the 21st century. Arch Surg, 142, 181-186.
Sharlf, I., \& Sargent, J. D. (2006). Association between television, movie, and ídeo game exposure and school performance. Pediatrics, 118(4), 1061-1070.

Sternheimer, K. (2007). Do ídeo games kill? Contexts, 6(1), 13-17.

Stickgold, R., Malia, A., Maguire, D., Roddenberry, D., \& O’Connor, M. (2000). Replayng the game: Hypnagogic images in normal and amnesics. Science, 290, 350-353.

Uhlmann, E., \& Swanson, J. (2004). Exposure to violent ídeo games increases automatic aggressiveness. International Journal of Adolescence and Youth, 27(1), 41-52.

Wang, X., \& Perry, A. C. (2006). Metabolic and physiologic responses to ídeo game play in 7-to 10-year-old boys. Arch pediatr adolesc med, 160, 411-415.

Widman, L. M., McDonald, C. M., \& Abresch, R. T. (2006). Effectiveness of na Upper Extremity Exercise Device Integrated With Computer Gaming for Aerobic Training in Adolescents With Spinal Cord Dysfunction. J. Spinal Cord Med., 29(4), 363370 .

Yoon, S. L., \& Godwin, A. (2007). Enhancing self-management in children with sickle cell disease through playing a CD-ROM educational game: A pilot study. Pediatric Nursing, 33(1), 60-63.

Zapata, A. L., Moraes, A. J. P., Leone, C., Doria-Filho, U., \& Silva, C.A.A. (2006). Pain and musculoskeletal pain syndromes related to computer and ídeo game use in adolescents. Eur J Pediatr, 165 , $408-414$.

Recebido em 06/10/2009 Aceito em 15/07/2011
Endereço para correspondência:
Luciana Alves. Rua São Paulo, 351/1403, Centro, CEP 30170-130, Belo HorizonteMG, Brasil. E-mail: lualbhmg@gmail.com. 